\title{
Chronic Dizziness and Mental Health Comorbidity
}

DOI: $10.3766 /$ jaaa.30.9.1

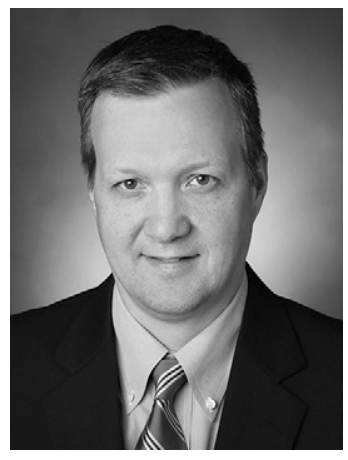

$\mathrm{T}$ he majority of patients who report experiencing an acute vestibular syndrome (i.e., neuritis or labyrinthine impairment) will be symptom free after a few weeks. There are those, however, who continue to experience symptoms even following medical treatment and therapy.

This is extremely frustrating to both the physician and the patient. In fact, it has been reported that approximately one quarter of patients develop a persistent dizziness disorder following the initial event. This statistic is in line with episodic refractory vestibular disorders (e.g., vestibular migraine and Menière's disease) and is even higher in those individuals who have incurred a mild traumatic brain injury (mTBI).

While each patient's set of symptoms may vary with chronic dizziness (i.e., non-vertiginous), they will often describe the symptoms as being continuously present throughout the day and the severity of the sensations may vary in intensity. They will also frequently describe the subjective sensation of an internal rocking or unsteadiness that they liken to being on a dock in a calm lake.

Patients with chronic symptoms of dizziness may also describe the sensation of pulsion or even report brief episodes of rotary vertigo. Another key feature often observed in patients that suffer from chronic dizziness is sensitivity to visual motion. Specifically, motion-rich environments, environments with complex visual patterns or situations during which they are moving their head, are particularly provocative. These symptoms have been shown to have a profound effect on the patient's quality of life and daily functioning.

When studies are done that examine the relationship between the severity of symptoms in patients with chronic dizziness and the extent of the peripheral vestibular impairments, the two are poorly correlated. This would seem to indicate that what is driving the persistent symptoms are higher-level brain processes and not the status of the peripheral structures.

This runs counter to previous theories that attempted to explain chronic dizziness as "chronic vestibulopathy" (CV). The driving theory behind CV was, for reasons that are unclear, that the compensation mechanisms of the vestibular system were impaired such that complete compensation for the insult could not be achieved.

While this concept was adopted for decades, new studies have found that the story may, in fact, be more complicated. Research over the last few decades have shown that, rather than simply being a case of impaired central nervous system compensation, distinct changes in the way the brain processes spatial information may be the primary contributor to the symptoms.

Specifically, the processes that govern the coordination of postural control and the integration of sensory information may be disrupted, which results in a continuation of these persistent symptoms.

There are a number of laboratories that have studied and described these chronic clinical entities that are debilitating to the patient. Terms such as visual vertigo, space-motion discomfort, and chronic subjective dizziness are just a few of them. More recently, a criterion was published for persistent postural perceptual dizziness (PPPD), a disorder characterized by many of the symptoms described above (Staab et al, 2017).

In this month's issue of the Journal of the American Academy of Audiology, Fox and colleagues, in their article titled "Dizziness, Vertigo, and Mental Health Comorbidity in Gulf War Veterans," tackle the problem of dizziness and mental health comorbidity in veterans diagnosed with Gulf War illness.

The investigators sampled 50 veterans and characterized their symptoms associated with dizziness and vertigo 
and catalogued their mental health comorbidities. This study illustrates how we must begin to look beyond just the structural impairments that are identified by laboratory testing and treat the patient as a whole.

I encourage you to read this well-designed study. I am excited to see research of this type and caliber being applied to patients with chronic dizziness.

Devin L. McCaslin, PhD Deputy Editor-in-Chief

\section{REFERENCES}

Staab JP, Eckhardt-Henn A, Horii A, Jacob R, Strupp M, Brandt T, Bronstein A. (2017) Diagnostic criteria for persistent postural-perceptual dizziness (PPPD): Consensus document of the Committee for the Classification of Vestibular Disorders of the Bárány Society. J Vestibul Res 27(4):191-208.

Thompson KJ, Goetting JC, Staab JP, Shepard NT. (2015) Retrospective review and telephone follow-up to evaluate a physical therapy protocol for treating persistent posturalperceptual dizziness: a pilot study. J Vestibul Res 25(2):97103. 